\title{
Convolution-Dominated Operators on Discrete Groups
}

\author{
Gero Fendler, Karlheinz Gröchenig and Michael Leinert
}

\begin{abstract}
We study infinite matrices $A$ indexed by a discrete group $G$ that are dominated by a convolution operator in the sense that $|(A c)(x)| \leq(a *|c|)(x)$ for $x \in G$ and some $a \in \ell^{1}(G)$. This class of "convolution-dominated" matrices forms a Banach-*-algebra contained in the algebra of bounded operators on $\ell^{2}(G)$. Our main result shows that the inverse of a convolution-dominated matrix is again convolution-dominated, provided that $G$ is amenable and rigidly symmetric. For abelian groups this result goes back to Gohberg, Baskakov, and others, for non-abelian groups completely different techniques are required, such as generalized $L^{1}$-algebras and the symmetry of group algebras.
\end{abstract}

Mathematics Subject Classification (2000). Primary 47B35; Secondary 43A20.

Keywords. Groups of polynomial growth, convolution, symmetric Banach algebras, inverse-closed, generalized $L^{1}$-algebra.

\section{Introduction}

Is the off-diagonal decay of an infinite matrix inherited by its inverse matrix? This question arises in many problems in numerical analysis and approximation theory and its solution has many applications in frame theory and pseudodifferential operators and wireless communications. See $[5,11,13,18,29,30]$ for a sample of papers.

The study of the off-diagonal decay has two distinct facets, namely the rate of the off-diagonal decay and the nature of the underlying index set. Usually the index set is (a subset of) $\mathbb{Z}^{d}$ and the focus is on obtaining various forms of offdiagonal decay conditions. For instance, it is known that polynomial decay and subexponential decay are preserved under matrix inversion [18, 15].

In general, the preservation of off-diagonal decay under inversion depends also on the index set. For instance, in the theory of Calderòn-Zygmund operators, 
the index set consists of all dyadic cubes. On this index set the quality of the off-diagonal decay is not necessarily preserved, and as a consequence the inverse of a Calderòn-Zygmund operator need not be a Calderòn-Zygmund operator [25, 32] .

Thus the interaction between the precise form of off-diagonal decay and the index set plays a decisive role. This observation is implicit in $[18,15,31]$. In $[18,15]$ it was mentioned (without explicit proof) that polynomial or subexponential decay are preserved under inversion whenever the index set of the matrix class possesses a metric with a polynomial growth condition.

We study the interaction between the decay conditions and the index set in the context of non-commutative harmonic analysis. Precisely, the index set will be a discrete (non-Abelian) group, e.g., a finitely generated discrete group of polynomial growth. We then investigate the class of convolution-dominated matrices, which are described by a specific type of off-diagonal decay. Convolutiondominated matrices over the index set $\mathbb{Z}^{d}$ were introduced by Gohberg, Kashoeck, and Woerdeman [10] as a generalization of Toeplitz matrices, and they showed that this class of matrices was closed under inversion. Similar results and generalizations were obtained independently by Kurbatov [19], Baskakov [2]. Sometime later Sjöstrand [29] rediscovered their results, gave a completely different proof, and used it in the context of a deep theorem about pseudodifferential operators.

We consider matrices indexed by a discrete group $G$ : every operator on $\ell^{2}(G)$ is described by a matrix $A$ with entries $A(x, y), x, y \in G$ by the usual action $(A c)(x)=\sum_{y \in G} A(x, y) c(y)$ on a sequence $c \in \ell^{2}(G)$. We will consider mostly groups of polynomial growth. A finitely generated group is of polynomial growth, if there exists a finite set $U \subseteq G$, such that $\bigcup_{n=1}^{\infty} U^{n}=G$ and card $U^{n} \leq C n^{D}$ for some constants $C, D>0$. Our main theorem reads as follows.

Theorem 1. Let $G$ be a discrete finitely generated group of polynomial growth. If a matrix $A$ indexed by $G$ satisfies the off-diagonal decay condition $|A(x, y)| \leq$ $a\left(x y^{-1}\right), x, y \in G$ for some $a \in \ell^{1}(G)$ and $A$ is invertible on $\ell^{2}(G)$, then there exists $b \in \ell^{1}(G)$ such that $\left|A^{-1}(x, y)\right| \leq b\left(x y^{-1}\right), x, y \in G$.

We will extend this result and also consider the situation where $\ell^{1}(G)$ is replaced by the weighted algebra $\ell^{1}(G, \omega)$ for certain weight functions on $G$. This weighted case is easier and follows from Theorem 1 by standard methods.

To put Theorem 1 into a bigger context, let us consider the case $A(x, y)=$ $a\left(x y^{-1}\right)$ for a sequence $a \in \ell^{1}(G)$. This matrix $A$ corresponds to the convolution operator $A c=a * c$ on $\ell^{2}(G)$. Even this case is highly non-trivial. Theorem 1 implies the symmetry of the group algebra $\ell^{1}(G)$, i.e., the spectrum of positive elements $a^{*} * a$ is contained in $[0, \infty)$ for all $a \in \ell^{1}(G)$. This fact is of course well known, but its proof requires the combination of two landmark results of harmonic analysis, namely Gromov's characterization of finitely generated groups of polynomial growth as finite extensions of nilpotent groups and Hulanicki's result that discrete nilpotent groups are symmetric $[16,17]$.

Convolution-dominated matrices on groups of polynomial growth occur implicitly in Sun's remarkable work [31]. His conditions on the off-diagonal decay 
are somewhat complicated and exclude the basic case of $\ell^{1}$-decay. In view of the relation with the symmetry of groups, this omission is not surprising.

If $G$ is a discrete Abelian group $G$, the proof of the main theorem is based on an idea of de Leeuw [4]: to every operator on $G$ one can assign an operatorvalued Fourier series and then classical Fourier series arguments, such as Wiener's Lemma, can be applied. This approach is championed in $[2,10]$.

For a non-Abelian group as the index set, these ideas break down completely, and a new approach is required. Our key idea is to replace the Fourier series arguments by methods taken from Leptin's investigation of generalized $L^{1}$ algebras $[20,21,22]$. The main insight is that the algebra of convolution-dominated matrices can be identified with a generalized $L^{1}$-algebra in the sense of Leptin. This observation allows us to translate the original problem about matrix inversion into a problem of abstract harmonic analysis. The analysis of generalized $L^{1}$-algebras was advanced by Leptin and Poguntke [20, 21, 22, 24] and has produced deep results. In fact, we will resort to their representation theoretic results and to the concept of the "rigid symmetry" of Banach algebras and apply these at a crucial point.

The relation between a "simple" matrix problem and the theory of generalized $L^{1}$-algebras may seem surprising at first glance, but it is exactly this connection that allows us to use the power of non-commutative harmonic analysis to solve the problem.

Let us mention that a similar theory can be established for convolution-dominated integral operators. This generalization is more technical and will be dealt with in a subsequent paper.

The paper is organized as follows: in Section 2 we give a formal definition of the algebra of convolution-dominated operators and identify it as a generalized $L^{1}$-algebra. In Section 3 we prove the symmetry of this algebra, and in Section 4 we treat the related concept of inverse-closedness. In particular, we prove Theorem 1. In Section 5 we treat the weighted case and characterize all weights for which the generalized weighted $L^{1}$-algebra is symmetric.

Acknowledgement: We would like to thank Marc Rieffel for his useful comments and questions on an early draft of the paper.

\section{The Algebra of Convolution-Dominated Operators as a Twisted $L^{1}$-Algebra}

Let $G$ be a discrete group. For $x \in G$ we denote the operator of left translation on $\ell^{1}(G)$ and on $\ell^{2}(G)$ by $\lambda(x)$, i.e. if $f \in \ell^{1}(G)$ or $f \in \ell^{2}(G)$, then $\lambda(x) f(y)=$ $f\left(x^{-1} y\right), x, y \in G$. By $\mathcal{B}\left(\ell^{2}(G)\right)$ we denote the algebra of bounded operators on $\ell^{2}(G)$.

For an operator $A: \ell^{2}(G) \rightarrow \ell^{2}(G)$ let $A(x, y)=<A \delta_{y}, \delta_{x}>, x, y \in G$, be its matrix, where $<$, $>$ is the inner product of the Hilbert space $\ell^{2}(G)$ and $\delta_{x}(x)=1$ and $\delta_{x}(z)=0$ for $z \neq x$. 
Definition 1. The operator $A$ is called convolution-dominated, in short notation $A \in C D(G)$, if there exists a sequence $a \in \ell^{1}(G)$ such that

$$
|A(x, y)| \leq a\left(x y^{-1}\right), \quad \forall x, y \in G .
$$

We define the norm of $A$ as an element in $C D(G)$ by

$$
\|A\|_{1}:=\inf \left\{\|a\|_{\ell^{1}}: a \in \ell^{1}(G),|A(x, y)| \leq a\left(x y^{-1}\right) \forall x, y \in G\right\} .
$$

By choosing $a(z)$ to be the supremum of the entries of $A$ on the $z$-th diagonal, namely $a(z)=\sup _{\left\{x, y: x y^{-1}=z\right\}}|A(x, y)|$, we see that

$$
\|A\|_{1}=\sum_{z \in G} \sup _{\left\{x, y: x y^{-1}=z\right\}}|A(x, y)|<\infty .
$$

To shed light on this definition, consider the action of the operator or matrix $A$ on a finitely supported vector $c$ and take absolute values:

$$
|(A c)(x)|=\left|\sum_{y \in G} A(x, y) c(y)\right| \leq \sum_{y \in G} a\left(x y^{-1}\right)|c(y)|=(a *|c|)(x) .
$$

Thus $A$ is dominated pointwise by a convolution operator, whence our terminology. Clearly, if $a \in \ell^{1}(G)$, i.e., if $A \in C D(G)$, then $A$ is bounded on $\ell^{2}(G)$, and the operator norm on $\ell^{2}(G)$, in fact on all $\ell^{p}(G), 1 \leq p \leq \infty$, is majorized by the $\|A\|_{1^{-}}$ norm. If we consider the composition of two convolution-dominated operators $A$ and $B$, then we obtain similarly

$$
|(A B c)(x)| \leq(a * b *|c|)(x),
$$

and therefore the operator $A B$ is again convolution-dominated and we obtain that $\|A B\|_{1} \leq\|A\|_{1}\|B\|_{1}$, because $\ell^{1}(G)$ is a convolution algebra. We may summarize these observations as follows.

Lemma 1. The space $C D(G)$ is a Banach *-algebra with respect to composition of operators and taking the adjoint operator as involution. Moreover, $C D(G)$ is continuously embedded into $\mathcal{B}\left(\ell^{2}(G)\right)$.

Our first goal is to represent $C D(G)$ as a generalized $L^{1}$-algebra in the sense of Leptin [20]. Consider the $C^{*}$-algebra $\ell^{\infty}(G)$ with pointwise multiplication and complex conjugation as involution. This algebra is isometrically represented as an algebra of multiplication operators on $\ell^{2}(G)$ by

$$
D^{m} f(x)=m(x) f(x) \text {, where } x \in G, f \in \ell^{2}(G), m \in \ell^{\infty}(G) .
$$

Analogously, we define an operator $D_{z}^{m}$ by

$$
D_{z}^{m}=\lambda(z) \circ D^{m} \text {. }
$$

As is easily seen, the matrix of $D_{z}^{m}$ has the entries

$$
D_{z}^{m}(x, y)=m(y) \delta_{z}\left(x y^{-1}\right) .
$$

Whereas the matrix of the multiplication operator $D^{m}$ is a diagonal matrix, the matrix of $D_{z}^{m}$ is non-zero only on the $z$-th side-diagonal. Since every matrix can be written as the sum of its side-diagonals, every operator is a sum of the elementary 
operators $D_{z}^{m}$. This simple observation is crucial for the analysis of convolutiondominated operators.

Next we study how the operators $D_{z}^{m}$ behave under composition: if $v, w \in G$ and $m, n \in \ell^{\infty}(G)$, then

$$
\begin{aligned}
\left(D_{v}^{n} \circ D_{w}^{m}\right)(x, y) & =\sum_{z \in G} D_{v}^{n}(x, z) D_{w}^{m}(z, y) \\
& =\sum_{z \in G} n(z) \delta_{v}\left(x z^{-1}\right) m(y) \delta_{w}\left(z y^{-1}\right) \\
& =\sum_{z \in G} n(z y) \delta_{v}\left(x y^{-1} z^{-1}\right) m(y) \delta_{w}(z) \\
& =n(w y) m(y) \delta_{v}\left(x y^{-1} w^{-1}\right) \\
& =n(w y) m(y) \delta_{v w}\left(x y^{-1}\right) \\
& \left.=D_{v w}^{\left(T_{w}-1\right.} n\right) m(x, y) .
\end{aligned}
$$

In the last equality we have set $T_{y} n(z)=n\left(y^{-1} z\right)$ for $n \in \ell^{\infty}(G)$. We use a notation different from $\lambda$, because $T_{y}: \ell^{\infty}(G) \rightarrow \ell^{\infty}(G)$ is a $C^{*}$ automorphism of the algebra $\ell^{\infty}(G)$ and the mapping $y \mapsto T_{y}$ defines a homomorphism of the group $G$ into the group of $C^{*}$-automorphisms of $\ell^{\infty}(G)$. Using this homomorphism, we may now form the twisted $L^{1}$-algebra $\mathcal{L}=\ell^{1}\left(G, \ell^{\infty}(G), T\right)$ in the sense of Leptin $[20,21,22]$. The underlying Banach space of $\mathcal{L}$ is the space of $\ell^{\infty}(G)$ valued absolutely summable sequences on $G$, but we will often interpret it as the projective tensor product

$$
\ell^{1}\left(G, \ell^{\infty}(G)\right)=\ell^{1}(G) \hat{\otimes} \ell^{\infty}(G) .
$$

Thus for an element $f \in \ell^{1}\left(G, \ell^{\infty}(G)\right)$ we denote its value in $\ell^{\infty}(G)$ by $f(x)$, $x \in G$, and we write $f(x)(z)$ or $f(x, z)$ for the value of this $\ell^{\infty}$-function at $z \in G$.

The twisted convolution of $h, f \in \mathcal{L}$ is defined by

$$
h \star f(x)=\sum_{y \in G} T_{y} h(x y) f\left(y^{-1}\right), \quad \text { for } x \in G,
$$

and the involution of $h \in \mathcal{L}$ by

$$
h^{*}(x)=\overline{T_{x^{-1}} h\left(x^{-1}\right)}, \text { for } x \in G .
$$

An element $f \in \mathcal{L}$. may be represented uniquely as

$$
f=\sum_{z \in G} m_{z} \delta_{z}
$$

where $m_{z}=f(z) \in \ell^{\infty}(G)$. By using $m_{z}$ as the $z$-th side-diagonal of a matrix, we define a map

by

$$
R: \ell^{1}\left(G, \ell^{\infty}(G), T\right) \rightarrow B\left(\ell^{2}(G)\right)
$$

$$
R f=\sum_{z \in G} D_{z}^{m_{z}}
$$


Proposition 1. The map $R: \ell^{1}\left(G, \ell^{\infty}(G), T\right) \rightarrow C D(G)$ is an isometric *-isomorphism.

Proof. Let $f=\sum_{z \in G} m_{z} \delta_{z}$ and $h=\sum_{z \in G} n_{z} \delta_{z} \in \mathcal{L}$. By (2) we have

$$
\begin{aligned}
\|R f\|_{C D} & =\left\|\sum_{z \in G} D_{z}^{m_{z}}\right\|_{1} \\
& =\sum_{z \in G} \sup _{\left\{x, y: x y^{-1}=z\right\}}\left|D_{z}^{m_{z}}(x, y)\right| \\
& =\sum_{z \in G} \sup _{y}\left|m_{z}(y)\right|=\|f\|_{\ell^{1}\left(G, \ell^{\infty}(G)\right)}
\end{aligned}
$$

Thus $R$ is an isometry.

The twisted convolution of $f$ and $h$ may be computed as follows:

$$
\begin{aligned}
(h \star f)(x, z) & =\left[\sum_{y \in G} T_{y} h(x y) f\left(y^{-1}\right)\right](z) \\
& =\sum_{y \in G} h\left(x y, y^{-1} z\right) f\left(y^{-1}, z\right) \\
& =\sum_{y \in G} n_{x y}\left(y^{-1} z\right) m_{y^{-1}}(z) \\
& =\sum_{v} l_{v}(z) \delta_{v}(x)
\end{aligned}
$$

where

$$
l_{v}=\sum_{y \in G} T_{y} n_{v y} m_{y^{-1}} \in \ell^{\infty}(G) .
$$

By comparison, the composition of the corresponding operators $R f$ and $R h$ (matrix multiplication) yields that

$$
\begin{aligned}
R f \circ R h & =\sum_{r} D_{r}^{n_{r}} \circ \sum_{w} D_{w}^{m_{w}} \\
& =\sum_{r, w} D_{r w}^{\left(T_{w}-1 n_{r}\right) m_{w}}=\sum_{v} D_{v}^{l_{v}^{\prime}}
\end{aligned}
$$

where

$$
l_{v}^{\prime}=\sum_{\{r, w: r w=v\}} T_{w^{-1}} n_{r} m_{w}=\sum_{y \in G} T_{y} n_{v y} m_{y^{-1}}=l_{v} .
$$

Thus $R f \circ R h=R(f \star h)$ and $R$ is an algebra homomorphism.

The involution of $f$ as above is given by

$$
\begin{aligned}
f^{*}(x, z) & =\overline{T_{x^{-1}} m_{x^{-1}}(z)} \\
& =\overline{m_{x^{-1}}(x z)} \\
& =\sum_{v} l_{v}(z) \delta_{v}(x),
\end{aligned}
$$


where $l_{v}(z)=\overline{T_{v^{-1}} m_{v^{-1}}}(z)$.

By comparison, the adjoint of a single side-diagonal operator is

$$
\begin{aligned}
\left(D_{v}^{m_{v}}\right)^{*}(x, y) & =\overline{D_{v}^{m_{v}}(y, x)} \\
& =\overline{m_{v}(x)} \delta_{v}\left(y x^{-1}\right) \\
& =\overline{m_{v}(x)} \delta_{v^{-1}}\left(x y^{-1}\right) \\
& =\overline{m_{v}\left(v^{-1} y\right)} \delta_{v^{-1}}\left(x y^{-1}\right) \\
& =D_{v^{-1}}^{T_{v} \overline{m_{v}}}(x, y) .
\end{aligned}
$$

These equalities imply that

$$
\begin{aligned}
\left(\sum_{v} D_{v}^{m_{v}}\right)^{*} & =\sum_{v} D_{v^{-1}}^{T_{v} \overline{m_{v}}} \\
& =\sum_{v} D_{v}^{T_{v-1} \overline{m_{v-1}}}=R\left(f^{*}\right),
\end{aligned}
$$

and so $R$ preserves the involution. Finally, from the definition of $\|A\|_{1}$ and the equalities (2) and (5) one sees that $R$ is onto.

\section{Symmetry of the Twisted $L^{1}$-Algebra}

Recall that a Banach algebra $A$ with isometric involution is called symmetric if the spectrum of every positive element is contained in the non-negative reals, i. e. $\operatorname{sp}\left(a^{*} a\right) \subset[0, \infty)$ for all $a \in A$. For various abstract characterizations of symmetry see [3, Section 41] or [24].

Furthermore, a locally compact group $G$ is called symmetric, if its convolution algebra $L^{1}(G)$ is symmetric. Various classes of groups are known to be symmetric: (a) locally compact Abelian groups, (b) compact groups, (c) finite extensions of discrete nilpotent groups, (d) compactly generated groups of polynomial growth, (e) compact extensions of locally compact nilpotent groups, and others. See [23]. For the groups of the classes (a) - (c) Leptin and Poguntke [24] have shown that they satisfy an even stronger property, namely that of rigid symmetry. This means that for every $C^{*}$-algebra $C$ the projective tensor product $L^{1}(G) \hat{\otimes} C$ is symmetric. Later Poguntke [27] showed that all nilpotent locally compact groups are rigidly symmetric.

Our goal is to show that the twisted $L^{1}$-algebra $\mathcal{L}=\ell^{1}\left(G, \ell^{\infty}(G), T\right)$ of a rigidly symmetric discrete group $G$ is symmetric and hence that the algebra of convolution-dominated operators $C D(G)$ is also symmetric.

To this end we define a map

$$
Q: \ell^{1}\left(G, \ell^{\infty}(G), T\right) \rightarrow \ell^{1}(G) \hat{\otimes} B\left(\ell^{2}(G)\right)
$$

by

$$
f=\sum_{v} \delta_{v} \otimes m_{v} \mapsto \sum_{v} \delta_{v} \otimes D_{v}^{m_{v}}
$$


Proposition 2. The map $Q$ is an isometric *-isomorphism of $\ell^{1}\left(G, \ell^{\infty}(G), T\right)$ onto a closed subalgebra of $\ell^{1}(G) \hat{\otimes} B\left(\ell^{2}(G)\right)$.

Proof. The proof rests on the isometrical identification $\ell^{1}(G, E)=\ell^{1}(G) \hat{\otimes} E$, which holds for any Banach space $E$ [6, Ch. VIII.1.]. It follows that for $f=$ $\sum_{v} \delta_{v} \otimes m_{v} \in \mathcal{L}$

$$
\begin{aligned}
\|f\|_{1} & =\sum_{v}\left\|m_{v}\right\|_{\infty}=\sum_{v}\left\|D_{v}^{m_{v}}\right\|_{B\left(\ell^{2}(G)\right)} \\
& =\left\|\sum_{v} \delta_{v} \otimes D_{v}^{m_{v}}\right\|_{\ell^{1}(G) \hat{\otimes} B\left(\ell^{2}(G)\right)} .
\end{aligned}
$$

Thus $Q$ is an isometry. Let $h=\sum_{v} \delta_{v} \otimes n_{v}$, then by (6)

$$
h \star f=\sum_{v} \delta_{v} \otimes l_{v},
$$

where $l_{v}=\sum_{y \in G}\left(T_{y} n_{v y}\right) m_{y^{-1}}$. Hence

$$
\begin{aligned}
Q(h \star f) & =\sum_{v} \delta_{v} \otimes D_{v}^{l_{v}} \\
& =\sum_{v} \delta_{v} \otimes \sum_{\{z, w: z w=v\}} D_{z}^{n_{z}} D_{w}^{m_{w}} \\
& =\sum_{z, w} \delta_{z} \delta_{w} \otimes D_{z}^{n_{z}} D_{w}^{m_{w}} \\
& =\left(\sum_{z \in G} \delta_{z} \otimes D_{z}^{n_{z}}\right)\left(\sum_{w} \delta_{w} \otimes D_{w}^{m_{w}}\right)=Q(h) Q(f) .
\end{aligned}
$$

Similarly one computes that $Q$ intertwines the involutions. In fact

$$
\begin{aligned}
Q(f)^{*} & =\sum_{v} \delta_{v}^{*} \otimes\left(D_{v}^{m_{v}}\right)^{*} \\
& =\sum_{v} \delta_{v^{-1}} \otimes D_{v^{-1}}^{T_{v} \overline{m_{v}}} \\
& =\sum_{v^{-1}} \delta_{v} \otimes D_{v}^{T_{v-1} \overline{m_{v}-1}}=Q\left(f^{*}\right) .
\end{aligned}
$$

Thus $Q$ is a *-homomorphism. Since $Q$ is an isometry, the image of $Q$ is a closed subalgebra of $\ell^{1} \hat{\otimes} \mathcal{B}\left(\ell^{2}\right)$.

Since symmetry is inherited by closed subalgebras, we obtain the following consequence.

Corollary 1. Let $G$ be a discrete rigidly symmetric group. Then $\ell^{1}\left(G, \ell^{\infty}(G), T\right)$ and $C D(G)$ are symmetric Banach $*$-algebras. 


\section{Inverse Closedness}

Given two Banach algebras $\mathcal{A} \subseteq \mathcal{B}$ with common identity, $\mathcal{A}$ is inverse-closed in $\mathcal{B}$, if

$$
a \in \mathcal{A} \text { and } a^{-1} \in \mathcal{B} \Rightarrow a^{-1} \in \mathcal{A} .
$$

This notion occurs under many names: one also says that $\mathcal{A}$ is a spectral subalgebra or a local subalgebra of $\mathcal{B}$. The pair $(\mathcal{A}, \mathcal{B})$ is called a Wiener pair by Naimark [26]. An important property of an inverse-closed subalgebra $\mathcal{A}$ is that it possesses the same holomorphic functional calculus as $\mathcal{B}$.

Inverse-closedness is usually proved by means of Hulanicki's Lemma [17]. Let $r_{\mathcal{A}}(a)$ denote the spectral radius of $a$ in the algebra $\mathcal{A}$. If $r_{\mathcal{A}}(a)=r_{\mathcal{B}}(a)$ for all $a=a^{*} \in \mathcal{A}$, then we have equality of the $\operatorname{spectra} \operatorname{sp}_{\mathcal{A}}(a)=\operatorname{sp}_{\mathcal{B}}(a)$ for all $a \in \mathcal{A}$. Consequently, if $\mathcal{B}$ is symmetric, then $\mathcal{A}$ is also symmetric. For this version of Hulanicki's lemma, see [9, Lemma 3.1 and 6.1] and [12, Lemma 5.1] for an elementary proof.

Our goal is to show that the algebra of convolution-dominated matrices $C D(G)$ is inverse-closed in $\mathcal{B}\left(\ell^{2}(G)\right)$. For this purpose we consider two natural unitary representations of the twisted $L^{1}$-algebra $\mathcal{L}$.

The first representation is the so-called $D$-regular representation of $\mathcal{L}$. Recall that $D: m \mapsto D^{m}$ is a faithful representation of the $C^{*}$-algebra $\ell^{\infty}$ by multiplication operators in $B\left(\ell^{2}(G)\right)$. Then as in Leptin [22, §3] the $D$-regular representation $\lambda^{D}$ of $\mathcal{L}=\ell^{1}\left(G, \ell^{\infty}, T\right)$ on the Hilbert space $\ell^{2}\left(G, \ell^{2}(G)\right)$ is defined by

$$
\lambda^{D}(f) \xi(x)=\sum_{y \in G} D^{T_{y} f(x y)} \xi\left(y^{-1}\right), \xi \in \ell^{2}\left(G, \ell^{2}(G)\right), f \in \mathcal{L} .
$$

One easily verifies that this defines indeed a $*$-representation of $\mathcal{L}$.

The second representation is the mapping $R: \mathcal{L} \rightarrow C D(G) \subset B\left(\ell^{2}(G)\right)$ introduced in (5). By Proposition $1, R$ is also a $*$-representation of $\mathcal{L}$ on $\ell^{2}(G)$. We call this representation the canonical representation of $\mathcal{L}$.

Proposition 3. The D-regular representation $\lambda^{D}$ of $\mathcal{L}$ is a multiple of the canonical representation $R$. Hence $\|R(f)\|=\left\|\lambda^{D}(f)\right\|$ for all $f \in \mathcal{L}$.

Proof. We identify $\ell^{2}\left(G, \ell^{2}(G)\right)$ with $\ell^{2}(G \times G)$. Let $R^{\omega}$ be the extension of $R$ from $\ell^{2}(G)$ to $\ell^{2}(G \times G)$ by letting the operators $R(f)=\sum_{y \in G} \lambda(y) \circ D^{f(y)}, f \in \mathcal{L}$, act in the first coordinate only, i.e., for $\xi \in \ell^{2}(G \times G)$

$$
R^{\omega}(f) \xi(x, z)=\sum_{y \in G} f(y)\left(y^{-1} x\right) \xi\left(y^{-1} x, z\right) .
$$

Next we define a candidate for an intertwining operator between the $D$-regular representation and the $\operatorname{card}(G)$-multiple $R^{\omega}$ of the canonical representation by

$$
S \xi(x, z)=\xi(x z, z) \text {, where } \xi \in \ell^{2}(G \times G) .
$$

Then on the one hand we have

$$
S\left[R^{\omega}(f) \xi\right](x, z)=\sum_{y \in G} f(y)\left(y^{-1} x z\right) \xi\left(y^{-1} x z, z\right) .
$$


On the other hand

$$
\begin{aligned}
\lambda^{D}(f)(S \xi)(x, z) & =\sum_{y \in G}\left(T_{y} f(x y)\right)(z)(S \xi)\left(y^{-1}, z\right) \\
& =\sum_{y \in G}\left(T_{x^{-1} y} f(y)\right)(z)(S \xi)\left(y^{-1} x, z\right) \\
& =\sum_{y \in G} f(y)\left(y^{-1} x z\right)(S \xi)\left(y^{-1} x, z\right) \\
& =\sum_{y \in G} f(y)\left(y^{-1} x z\right) \xi\left(y^{-1} x z, z\right) .
\end{aligned}
$$

Consequently,

$$
\lambda^{D}(f)(S \xi)=S R^{\omega}(f) \xi
$$

for all $f \in \mathcal{L}$ and $\xi \in \ell^{2}(G \times G)$. Since $S$ is unitary on $\ell^{2}(G \times G), \lambda^{D}$ and $R^{\omega}$ are equivalent.

To deal with inverse-closedness, we need to compare several norms on $\mathcal{L}$ and $C D(G)$. Let $\|$. $\|_{*}$ be the largest $C^{*}$ norm on $\mathcal{L}$. By a theorem of Ptak [28] a Banach *-algebra $\mathcal{A}$ is symmetric, if and only if the largest $C^{*}$-seminorm $\|\cdot\|_{*}$ on $\mathcal{A}$ satisfies $\left\|a^{*} a\right\|_{*}=r_{\mathcal{A}}\left(a^{*} a\right)$ for all $a \in \mathcal{A}$. See also [3, $§ 41$ Corollary 8].

As a first consequence of Proposition 3 we identify the largest $C^{*}$-norm on $C D(G)$.

Corollary 2. Let $G$ be an amenable discrete group, then the largest $C^{*}$ norm on $\mathcal{L}$ equals the operator norm on $C D(G)$.

Proof. Since $G$ is amenable, it follows from [22, Satz 6] of Leptin that for the representation $D$ of $\ell^{\infty}(G)$ the $D$-regular representation $\lambda^{D}$ defines the largest $C^{*}$ norm on $\mathcal{L}$. Therefore we obtain

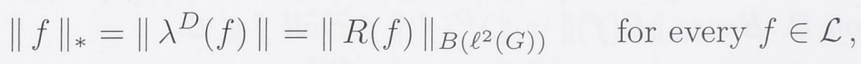

where the last equality follows from Proposition 3.

Proposition 4. Let $G$ be a discrete, amenable, and rigidly symmetric group. Then

$$
r_{\mathcal{L}}\left(f^{*} f\right)=r_{C D(G)}\left(R(f)^{*} R(f)\right)=\|R(f)\|_{B\left(L^{2}(G)\right)}^{2} \quad \text { for all } f \in \mathcal{L} .
$$

Proof. Since $\mathcal{L}$ and $C D(G)$ are symmetric by Corollary 1, Ptaks theorem [28] implies that $\|f\|_{*}^{2}=r_{\mathcal{L}}\left(f^{*} f\right)=r_{C D(G)}\left(R(f)^{*} R(f)\right)$. Since Corollary 2 says that $\left\|f^{*} f\right\|_{*}=\left\|R(f)^{*} R(f)\right\|_{\mathcal{B}\left(\ell^{2}\right)}$, we obtain the identity (11).

Theorem 2. Let $G$ be a discrete, amenable, and rigidly symmetric group. If $f \in \mathcal{L}$ is such that $R(f) \in C D(G)$ has an inverse in $B\left(\ell^{2}(G)\right)$ then $f^{-1}$ exists in $\mathcal{L}$ and $R\left(f^{-1}\right)=R(f)^{-1}$ is in $C D(G)$. 
Proof. If $f \in \mathcal{L}$ is hermitian, i.e. $f=f^{*}$, then by Proposition 4

$$
r_{\mathcal{L}}(f)^{2}=r_{\mathcal{L}}\left(f^{*} f\right)=\|R(f)\|_{B\left(L^{2}(G)\right)}^{2} .
$$

[9, Lemma 6.1 and 3.1] imply that

$$
s p_{\mathcal{L}}(f)=s p_{B\left(\ell^{2}(G)\right)}(R(f)), \quad \forall f \in \mathcal{L} .
$$

Thus the invertibility of $R(f)$ in $\mathcal{B}\left(\ell^{2}(G)\right)$ implies the invertibility of $f$ in $\mathcal{L}$.

By writing Theorem 2 explicitly as a statement about the off-diagonal decay of an invertible matrix, we recover Theorem 1 of the introduction.

Corollary 3. Let $G$ be a discrete, amenable, and rigidly symmetric group (for instance, a finitely generated group of polynomial growth). If a matrix $A$ indexed by $G$ satisfies the off-diagonal decay condition $|A(x, y)| \leq a\left(x y^{-1}\right)$ for some $a \in \ell^{1}(G)$ and $A$ is invertible on $\ell^{2}(G)$, then there exists $b \in \ell^{1}(G)$ such that $\left|A^{-1}(x, y)\right| \leq$ $b\left(x y^{-1}\right)$.

A slight variation yields the following result of which previous versions have been quite useful in time-frequency analysis [7].

Corollary 4. Assume that $A \in C D(G)$ and that $A=A^{*}$. Then the following are equivalent:

(i) $A$ is invertible on $\ell^{2}(G)$.

(ii) $A$ is invertible on $\ell^{p}(G)$ for all $p, 1 \leq p \leq \infty$.

(iii) $A$ is invertible on $\ell^{p}(G)$ for some $p, 1 \leq p \leq \infty$.

Proof. (i) $\Rightarrow$ (ii) Recall that every matrix $A \in C D(G)$ is bounded on all $\ell^{p}(G)$, $1 \leq p \leq \infty$ by (1). Thus if $A \in C D(G)$ is invertible on $\ell^{2}(G)$, then by Theorem 1 $A^{-1} \in C D(G)$ and thus $A^{-1}$ is invertible on $\ell^{p}(G)$ for arbitrary $p, 1 \leq p \leq \infty$. The implication (ii) $\Rightarrow$ (iii) is obvious.

(iii) $\Rightarrow$ (i) Assume that $A$ is invertible on some $\ell^{p}(G)$. Then the adjoint operator $A^{*}=A$ is invertible on the dual space $\ell^{p^{\prime}}(G)$, where $p^{\prime}=p /(p-1)$ is the conjugate index. By interpolation we obtain that $A$ is invertible on the interpolation space $\ell^{2}(G)$.

Remark. The hypotheses on the group $G$ are almost sharp. To see this, let $\lambda(f)$ denote the convolution operator $c \mapsto \lambda(f) c=f * c$ acting on $\ell^{p}(G)$, and let $s p_{\ell^{p}}(f)$ the spectrum of $\lambda(f)$ as an operator acting on $\ell^{p}(G)$. Then $\operatorname{sp}_{\ell^{p}}(f)=\operatorname{sp}_{\ell^{2}}(f)$ for all $p \in[1, \infty]$, if and only if the group $G$ is amenable and symmetric $[1,14]$. Thus amenability and symmetric are necessary in Theorem 2 . We do not know whether we can replace the rigid symmetry by symmetry, because it is an open problem whether every symmetric group is rigidly symmetric [27].

We emphasize once more that all discrete finitely generated groups of polynomial growth satisfy the hypotheses of amenability and rigid symmetry. These groups are finite extensions of some discrete nilpotent group by Gromov's result [16], and thus they are rigidly symmetric by [24] and [27]. 


\section{Symmetry of weighted algebras}

In this section we extend the results about the symmetry of convolution-dominated operators to the weighted case.

A function $\omega: G \rightarrow[1, \infty)$ is called a weight on $G$, if it fulfills

$$
\begin{aligned}
\omega(x y) & \leq \omega(x) \omega(y), \quad \forall x, y \in G \\
\omega\left(x^{-1}\right) & =\omega(x), \quad \forall x \in G \\
\omega(e) & =1 .
\end{aligned}
$$

Given a weight $\omega$ we let $\ell^{1}(G, \omega)$ be the weighted $\ell^{1}$-algebra on $G$. Using weights, one can model stronger decay conditions on convolution-dominated operators as follows.

Definition 2. An operator $A$ on $\ell^{2}(G)$ is called $\omega$-convolution-dominated, $A \in$ $C D(G, \omega)$ in short, if there exists an $a \in \ell^{1}(G, \omega)$ such that

$$
|A(x, y)| \leq a\left(x y^{-1}\right), \quad \forall x, y \in G .
$$

We define its norm as

$$
\|A\|_{\omega}:=\inf \left\{\|a\|_{\ell^{1}(G, \omega)}: a \in \ell^{1}(G, \omega),|A(x, y)| \leq a\left(x y^{-1}\right) \forall x, y \in G\right\} .
$$

As in the unweighted case, we may write the norm as

$$
\|A\|_{\omega}=\sum_{z \in G} \sup _{\left\{x, y: x y^{-1}=z\right\}}|A(x, y)| \omega(z)<\infty .
$$

Thus an operator $A$ is in $C D(G, \omega)$, if it is dominated by a convolution operator in $\ell^{1}(G, \omega)$ in the sense that $|A c(x)| \leq(a *|c|)(x)$ for some $a \in \ell^{1}(G, \omega)$. Since $\ell^{1}(G, \omega)$ is a convolution algebra, the space of $\omega$-convolution-dominated operators $C D(G, \omega)$ is a Banach *-algebra with respect to composition of operators and the usual involution of operators in $B\left(\ell^{2}(G)\right)$. Furthermore, $C D(G, \omega) \subseteq C D(G) \subseteq$ $\mathcal{B}\left(\ell^{2}(G)\right)$.

For the study of $C D(G, \omega)$, we consider the weighted, twisted $L^{1}$-algebra $\mathcal{L}_{\omega}=\ell^{1}\left(G, \omega, \ell^{\infty}(G), T\right)$, which is defined as a subalgebra of $\mathcal{L}$ endowed with the norm

$$
\|f\|_{\mathcal{L}_{\omega}}=\sum_{x \in G}\|f(x)\|_{\infty} \omega(x) .
$$

Since $\mathcal{L}_{\omega}$ is a subalgebra of $\mathcal{L}$, all algebraical relations are preserved and the results of Sections 2 and 3 carry over to $\mathcal{L}_{\omega}$ after a slight modification of the norm computations.

Proposition 5. Let $R_{\omega}$ and $Q_{\omega}$ denote the restrictions of the maps $R$ and $Q$ defined in (4) and (7) from $\mathcal{L}$ to $\mathcal{L}_{\omega}=\ell^{1}\left(G, \omega, \ell^{\infty}(G), T\right)$. Then

$$
R_{\omega}: \ell^{1}\left(G, \omega, \ell^{\infty}(G), T\right) \rightarrow C D(G, \omega)
$$

is an isometric *-isomorphism and

$$
Q_{\omega}: \ell^{1}\left(G, \omega, \ell^{\infty}(G), T\right) \rightarrow \ell^{1}(G, \omega) \hat{\otimes} B\left(\ell^{2}(G)\right)
$$

is an isometric *-isomorphism onto a closed *-subalgebra. 
We are interested in the symmetry of the weighted $\ell^{1}$-algebra. This forces us to impose some conditions of subexponential growth on the weight.

Definition 3. (a) A weight $\omega$ is said to satisfy the GRS-condition (GelfandRaikov-Shilov condition) if

$$
\lim _{n \rightarrow \infty} \omega\left(x^{n}\right)^{1 / n}=1 \quad \text { for all } x \in G .
$$

(b) A weight $\omega$ is said to satisfy the UGRS-condition (the uniform GRS-condition), if for some generating subset $U$ of $G$ containing the identity element

$$
\lim _{n \rightarrow \infty} \sup _{y \in U^{n}} \omega(y)^{1 / n}=\lim _{n \rightarrow \infty} \sup _{x_{1}, \ldots, x_{n} \in U} \omega\left(x_{1} x_{2} \ldots x_{n}\right)^{1 / n}=1 .
$$

The GRS-condition is a necessary condition for the spectral identity $r_{\ell^{1}}(f)=$ $r_{\ell_{\omega}^{1}}(f)$ in weighted group algebras, and hence for the symmetry of $\ell^{1}(G, \omega)$ [8]. If $G$ is a compactly generated locally compact group of polynomial growth, then the GRS-condition is also sufficient for the symmetry of $\ell^{1}(G, \omega)$. In this case, the UGRS-condition with a relatively compact set $U$ is also equivalent to the GRScondition by the results in [8]. However, if $G$ is not compactly generated, the UGRS-condition may be a stronger assumption on the weight.

We emphasize that in Definition 3, $U$ need not be finite. As a example consider the group $\mathbb{Z}^{2}$ and the weight $\omega\left(k_{1}, k_{2}\right)=\left(1+\left|k_{1}\right|\right)^{s}, k_{1}, k_{2} \in \mathbb{Z}, s>0$. This weight satisfies the GRS-condition and the UGRS-condition with the generating set $\{-1,0,1\} \times \mathbb{Z}$.

Theorem 3. Let $G$ be a rigidly symmetric, amenable, discrete group. If the weight $\omega$ satisfies the UGRS-condition and the condition

$$
\sup _{x \in U^{n} \backslash U^{n-1}} w(x) \leq C \inf _{x \in U^{n} \backslash U^{n-1}} w(x),
$$

then $\ell^{1}(G, \omega) \hat{\otimes} B\left(\ell^{2}(G)\right)$ is inverse-closed in $\ell^{1}(G) \hat{\otimes} \mathcal{B}\left(\ell^{2}(G)\right)$ and hence symmetric.

Proof. By the assumption on $G$ we know that the algebra $\mathcal{B}=\ell^{1}(G) \hat{\otimes} B\left(\ell^{2}(G)\right)$ is symmetric. Since $\mathcal{A}=\ell^{1}(G, \omega) \hat{\otimes} B\left(\ell^{2}(G)\right)$ is a subalgebra of $\mathcal{B}$, by $[9$, Lemmas 3.1 and 6.1$]$, we need only show the equality of the both spectral radii on the latter algebra.

Since for $f \in \mathcal{A}$

$$
\|f\|_{\mathcal{B}}=\sum_{x \in G}\|f(x)\|_{B\left(\ell^{2}(G)\right)} \leq \sum_{x \in G}\|f(x)\|_{B\left(\ell^{2}(G)\right)} \omega(x)=\|f\|_{\mathcal{A}},
$$

the spectral radius formula implies that

$$
r_{\mathcal{B}}(f) \leq r_{\mathcal{A}}(f) \quad \text { for all } f \in \mathcal{A} .
$$

Thus it suffices to show the converse inequality. To this end we define a weight $v$ on $\mathbb{Z}$ by

$$
v(n)=\sup _{y \in U|n|} \omega(y)
$$


where $U$ is a generating set, containing the identity element, such that $\lim _{n \rightarrow \infty} \sup _{y \in U^{n}} \omega(y)^{1 / n}=1$. By induction one finds an estimation for the norm of the n-th convolution power $f^{(n)}$ of $f \in \mathcal{A}$ :

$$
\left\|f^{(n)}\right\|_{\mathcal{A}} \leq \sum_{G} \cdots \sum_{G}\left\|f\left(x_{1}\right)\right\|\left\|f\left(x_{2}\right)\right\| \ldots\left\|f\left(x_{n}\right)\right\| \omega\left(x_{1} \ldots x_{n}\right) .
$$

Since $G=\bigcup_{n=1}^{\infty}\left(U^{n} \backslash U^{n-1}\right)$ as a disjoint union (where $U^{0}=\emptyset$ ), we may split each sum accordingly. This yields

$$
\begin{aligned}
& \left\|f^{(n)}\right\|_{\mathcal{A}} \leq \\
& \leq \sum_{k_{1}, k_{2}, \ldots, k_{n}=1}^{\infty} \sum_{U^{k_{1}} \backslash U^{k_{1}-1}} \ldots \sum_{U^{k_{n}} \backslash U^{k_{n}-1}}\left\|f\left(x_{1}\right)\right\| \ldots\left\|f\left(x_{n}\right)\right\| \omega\left(x_{1} \ldots x_{n}\right) .
\end{aligned}
$$

If $x_{j} \in U^{k_{j}} \backslash U^{k_{j}-1}$, then $x_{1} \ldots x_{n} \in U^{k_{1}+\cdots+k_{n}}$ and so the weight is majorized by

$$
\omega\left(x_{1} \ldots x_{n}\right) \leq \sup _{y \in U^{k_{1}+\cdots+k_{n}}} \omega(y)=v\left(k_{1}+\cdots+k_{n}\right) .
$$

Set $b_{k}:=\sum_{U^{k} \backslash U^{k-1}}\|f(x)\|$ and $b=\left(b_{k}\right)_{k \in \mathbb{N}}$. Then clearly we have $\|f\|_{\mathcal{B}}=\|b\|_{\ell^{1}}$ and condition (12) implies that $C^{-1}\|b\|_{\ell_{v}^{1}} \leq\|f\|_{\mathcal{L}_{\omega}} \leq\|b\|_{\ell_{v}^{1}}$. For the convolution powers of $f$ we obtain that

$$
\left\|f^{(n)}\right\|_{\mathcal{A}} \leq \sum_{k_{1}, k_{2}, \ldots, k_{n}=1}^{\infty} b_{k_{1}} b_{k_{2}} \ldots b_{k_{n}} v\left(k_{1}+k_{2}+\ldots k_{n}\right)=\left\|b^{(n)}\right\|_{\ell^{1}(\mathbb{Z}, v)}<\infty .
$$

By its definition the weight $v$ on $\mathbb{Z}$ satisfies the GRS-condition, and $\ell^{1}(\mathbb{Z}, v)$ is symmetric by [8, Lemma 3.2]. Hence

$$
\begin{aligned}
r_{\mathcal{A}}(f) & =\lim _{n \rightarrow \infty}\left\|f^{(n)}\right\|_{\mathcal{A}}^{1 / n} \leq \lim _{n \rightarrow \infty}\left\|b^{(n)}\right\|_{\ell^{1}(\mathbb{Z}, v)}^{1 / n} \\
& =r_{\ell^{1}(\mathbb{Z}, v)}(b)=r_{\ell^{1}(\mathbb{Z})}(b)=\|b\|_{\ell^{1}} \\
& =\|f\|_{\mathcal{B}} .
\end{aligned}
$$

So for all $k \in \mathbb{N}$ we have

$$
r_{\mathcal{A}}(f)=r_{\mathcal{A}}\left(f^{(k)}\right)^{1 / k} \leq\left\|f^{(k)}\right\|_{\mathcal{B}}^{1 / k},
$$

and by letting $k \rightarrow \infty$ we obtain the required inequality $r_{\mathcal{A}}(f) \leq r_{\mathcal{B}}(f)$.

Combining Proposition 5 and Theorem 3, we obtain the symmetry of the weighted convolution-dominated operator algebras $C D(G, \omega)$.

Corollary 5. Under the same assumptions on $G$ and $\omega$ as in Theorem 3, the algebra $C D(G, \omega)$ is symmetric.

Moreover, the Theorem 3 combined with Theorem 2 shows that for $f \in \mathcal{L}_{\omega}$ :

$$
\begin{aligned}
r_{\mathcal{L}_{\omega}}(f) & =r_{\mathcal{A}}\left(Q_{\omega}(f)\right)=r_{\mathcal{B}}(Q(f)) \\
& =r_{\mathcal{L}}(f)=r_{B\left(\ell^{2}(G)\right)}(R(f))=r_{B\left(\ell^{2}(G)\right)}\left(R_{\omega}(f)\right) .
\end{aligned}
$$

Using Hulanicki's Lemma in the form of [9, Lemma 6.1 and 3.1] we conclude as in the proof of Theorem 2 that $C D(G, \omega)$ is inverse-closed in $B\left(\ell^{2}(G)\right)$. 
Corollary 6. Impose the same assumptions on $G$ and $\omega$ as in Theorem 3.

If $f \in \mathcal{L}_{\omega}$ is such that $R_{\omega}(f) \in C D(G, \omega)$ has an inverse in $B\left(\ell^{2}(G)\right)$ then $f^{-1}$ exists in $\mathcal{L}_{\omega}$, and $R_{\omega}\left(f^{-1}\right)=R_{\omega}(f)^{-1}$ is in $C D(G, \omega)$.

For a single matrix Corollary 6 can be recast once again as a statement about the preservation of the off-diagonal decay by the inverse.

Corollary 7. Impose the same assumptions on $G$ and $\omega$ as in Theorem 3 . If a matrix $A$ on $G$ satisfies the off-diagonal decay condition $|A(x, y)| \leq a\left(x y^{-1}\right), \forall x, y \in G$, for some $a \in \ell^{1}(G, \omega)$ and $A$ is invertible on $\ell^{2}(G)$, then there exists some $b \in \ell^{1}(G, \omega)$, such that $\left|A^{-1}(x, y)\right| \leq b\left(x y^{-1}\right), \forall x, y \in G$.

Remark. The proof of Thm. 3 is similar to the one of [8, Thm. 3.3]. However, the proof given there works only under an additional assumption on the weight, such as (12), the result remains correct as a consequence of the main result in [8].

\section{References}

[1] B. A. Barnes. When is the spectrum of a convolution operator on $L^{p}$ independent of $p$ ? Proc. Edinburgh Math. Soc. (2), 33(2):327-332, 1990.

[2] A. G. Baskakov. Wiener's theorem and asymptotic estimates for elements of inverse matrices. Funktsional. Anal. i Prilozhen., 24(3):64-65, 1990.

[3] F. Bonsall and J. Duncan. Complete normed algebras. Springer Verlag, 1973.

[4] K. deLeeuw. An harmonic analysis for operators. I. Formal properties. Illinois J. Math., 19(4):593-606, 1975.

[5] S. Demko, W. F. Moss, and P. W. Smith. Decay rates for inverses of band matrices. Math. Comp., 43(168):491-499, 1984.

[6] J. Diestel, J. J. Uhl. Vector Measures. Math. Surveys 15, Amer. Math. Soc., Providence, RI, 1977.

[7] H. G. Feichtinger and K. Gröchenig. Gabor frames and time-frequency analysis of distributions. J. Functional Anal., 146(2):464-495, 1997.

[8] G. Fendler, K. Gröchenig, and Leinert. Symmetry of weighted $L^{1}$-algebras and the GRS-condition. Bull. London Math. Soc., 38:625-635, 2006.

[9] G. Fendler, K. Gröchenig, M. Leinert, J. Ludwig, and C. Molitor-Braun. Weighted group algebras on groups of polynomial growth. Math. Z., 245:791-821, 2003.

[10] I. Gohberg, M. A. Kaashoek, and H. J. Woerdeman. The band method for positive and strictly contractive extension problems: an alternative version and new applications. Integral Equations Operator Theory, 12(3):343-382, 1989.

[11] K. Gröchenig. Localization of frames, Banach frames, and the invertibility of the frame operator. J. Fourier Anal. Appl., 10(2), 2004.

[12] K. Gröchenig. Composition and spectral invariance of pseudodifferential operators on modulation spaces. J. Anal. Math., 98:65-82, 2006.

[13] K. Gröchenig. Time-frequency analysis of Sjöstrand's class. Revista Mat. Iberoam., 22(2):703-724, 2006. 
[14] K. Gröchenig and M. Leinert. Wiener's lemma for twisted convolution and Gabor frames. J. Amer. Math. Soc., 17:1-18, 2004.

[15] K. Gröchenig and M. Leinert. Symmetry and inverse-closedness of matrix algebras and functional calculus for infinite matrices. Trans. Amer. Math. Soc., 358(6):26952711 (electronic), 2006.

[16] M. Gromov. Groups of polynomial growth and expanding maps. Inst. Hautes Études Sci. Publ. Math., 53(1):53-78, 1981.

[17] A. Hulanicki. On the spectrum of convolution operators on groups with polynomial growth. Invent. Math., 17:135-142, 1972.

[18] S. Jaffard. Propriétés des matrices "bien localisées" près de leur diagonale et quelques applications. Ann. Inst. H. Poincaré Anal. Non Linéaire, 7(5):461-476, 1990.

[19] V. G. Kurbatov. Algebras of difference and integral operators. Funktsional. Anal. $i$ Prilozhen., 24(2):87-88, 1990.

[20] H. Leptin. Verallgemeinerte $L^{1}$-Algebren und projektive Darstellungen lokal kompakter Gruppen I. Invent. Math., 3:257-281, 1967.

[21] H. Leptin. Verallgemeinerte $L^{1}$-Algebren und projektive Darstellungen lokal kompakter Gruppen II. Inventiones Math., 4:68-86, 1967.

[22] H. Leptin. Darstellungen verallgemeinerter $L^{1}$-Algebren. Inventiones Math., pages 192-215, 1968.

[23] H. Leptin. The structure of $L^{1}(G)$ for locally compact groups. In Operator algebras and group representations, Vol. II (Neptun, 1980), volume 18 of Monogr. Stud. Math., pages 48-61. Pitman, Boston, MA, 1984.

[24] H. Leptin and D. Poguntke. Symmetry and non-symmetry for locally compact groups. J. Funct. Anal., 33:119-134, 1979.

[25] Y. Meyer. Ondelettes et opérateurs. II. Hermann, Paris, 1990. Opérateurs de Calderón-Zygmund. [Calderón-Zygmund operators].

[26] M. A. Naĭmark. Normed algebras. Wolters-Noordhoff Publishing, Groningen, third edition, 1972. Translated from the second Russian edition by Leo F. Boron, WoltersNoordhoff Series of Monographs and Textbooks on Pure and Applied Mathematics.

[27] D. Poguntke. Rigidly symmetric $L^{1}$-group algebras. Seminar Sophus Lie, 2:189-197, 1992.

[28] V. Pták. On the spectral radius in Banach algebras with involution. Bull. London Math. Soc., 2:327-334, 1970.

[29] J. Sjöstrand. Wiener type algebras of pseudodifferential operators. In Séminaire sur les Équations aux Dérivées Partielles, 1994-1995, Exp. No. IV, 19 pages. École Polytech., Palaiseau, 1995.

[30] T. Strohmer. Pseudodifferential operators and Banach algebras in mobile communications. Appl. Comput. Harmon. Anal., 20(2):237-249, 2006.

[31] Q. Sun. Wiener's lemma for infinite matrices. Trans. Amer. Math. Soc., 359(7):3099 3123 (electronic), 2007.

[32] P. Tchamitchian. Calcul symbolique sur les opérateurs de Calderón-Zygmund et bases inconditionnelles de $L^{2}(\mathbf{R})$. C. R. Acad. Sci. Paris Sér. I Math., 303(6):215218,1986 . 
Gero Fendler

Finstertal 16

D-69514 Laudenbach

Germany

e-mail: gero.fendler@t-online.de

Karlheinz Gröchenig

Fakultät für Mathematik

Universität Wien

Nordbergstrasse 15

A-1090 Wien

Austria

e-mail: karlheinz.groechenig@univie.ac.at

Michael Leinert

Institut für Angewandte Mathematik

Universität Heidelberg

Im Neuenheimer Feld 294

D-69120 Heidelberg

Germany

e-mail: leinert@math. uni-heidelberg.de

Submitted: January 2, 2008. 